\title{
PROGRESS IN PARTICLE IDENTIFICATION BY IONIZATION SAMPLING
}

\author{
I. LEHRAUS \\ CERN, Geneva, Suitzerland
}

The present generation of large scale $\mathrm{d} E / \mathrm{d} x$ detectors was conceived in the mid-seventies. In fixed target physics applications (EPI. ISIS) the achieved particle identification efficiency allows for $\pi / \mathrm{K}$ separation in the relativistic rise region. In the family of compact colliding beam detectors (e.g. AFS, ARGUS, CLEO, JADE, TPC, UAl), only the LBL TPC performance was so far sufficient for operation beyond the minimum of ionization. New central detectors foreseen for experiments in near future (LEP) should be more performing and will require resolutions which are at the limits imposed by systematic higher order effects. Influence of various design parameters on the optimization of the $\mathrm{d} E / \mathrm{d} x$ sampling performance is discussed, supported by recent result from measurements of full-scale detector segments and including tests using ionized tracks generated by pulsed UV lasers. Merits of longitudinal drift and fine sampling are compared to results obtained by classical charge integration.

\section{Introduction}

Particle identification by ionization sampling represents an attractive method for applications in compact colliding beam detectors, where it is easily combined with the track reconstruction task of the vertex chambers. The progress in the development of this method can be illustrated by examples of the history of several large scale devices, fully or at least partially dedicated to $\mathrm{d} E / \mathrm{d} x$ sampling. First detectors designed for hybrid bubble chamber experiments at fixed target accelerators were of a "box" geometry with 2-coordinate readout. Particle momenta were measured "outside", and the tracks inside the detector were straight (neglecting the influence of weak stray magnetic fields). Due to short beam spill and small duty cycle requirements of bubble chambers, the background level and corresponding space-charge problems were not too severe. The EPI detector for BEBC at CERN was proposed in 1973 [1], the construction started in 1974 [2] and the first data were taken with the fully operational set-up in 1978-1979 [3,4]. The EPI consists of a fixed matrix of rectangular proportional cells. Large drift volumes were introduced into the design of ISIS [5], proposed in 1974 for EHS at CERN. Full scale operation and data taking using ISIS started in 1980-1981 [6,7]. A reduced version of ISIS (CRISIS [8]) was proposed for FNAL hybrid experiment in 1978. Both EPI and ISIS are large volume atmospheric pressure detectors aiming at $\mathrm{d} E / \mathrm{d} x$ resolution near $6 \%$ fwhm, which is necessary for efficient particle identification up to about $100 \mathrm{GeV} / c$.

Colliding beam detectors operate in magnetic fields and must be more compact than the above mentioned devices. If the required resolution cannot be reached at atmospheric pressure in a given volume, the gas pressure must be correspondingly increased. The most ambitious design of a 3-dimensional pictorial chamber containing large drift space - TPC - was proposed in 1976 for PEP at SLAC [9]. This high pressure chamber took first data in $1982[10,11]$ and resolution comparable to EPI and ISIS performance was attained. Due to increased pressure the TPC cannot compete with the atmospheric pressure devices at very high particle momenta. The jet chamber for the JADE [12] experiment at PETRA was proposed in 1976 and the data taking started in 1980 [13]. In this detector the "bicycle wheel" geometry having moderate drift distances was adopted for the first time. A similar geometry was used for the AFS vertex detector at ISR (proposal in 1979 [14], completed in 1981-1982 [15]. The $\mathrm{d} E / \mathrm{d} x$ segments in the CLEO detector for CESR [16] were fully equipped in 1981-1982 [17]. Planar chambers with relatively long drift path were used in the design of the UAl central detector (dipole magnet) proposed in 1978 for the CERN $\bar{p} p$ collider [18], first data were taken in 1982 [19].

Basically, from the quoted examples we see that the presently operational generation of large scale detectors using $\mathrm{d} E / \mathrm{d} x$ sampling for particle identification was conceived in the mid- or late-seventies and that it took on the average about 4 to 5 years from prototype tests to full operation. A new generation of central detectors with improved performance will be needed in near future for experiments planned for LEP, SLC and TRISTAN. In this perspective, the possibilities and limits of the present state of the ionization sampling method will be examined in the following paragraphs, with emphasis put on the prospects of the $\pi / K$ separation at highest attainable momenta. More detailed knowledge about developments over the last few years may be obtained by comparing the relevant contributions to the 1980 
Uppsala Conference on Experimentation at LEP [20], to the 1982 SLAC Conference on Instrumentation for colliding beam physics [21] and to the 1982 Nuclear science Symposium [22].

\section{High resolution ionization sampling}

Fluctuations of the ionization energy loss in thin absorbers and their influence on the detector design were extensively treated theoretically and experimentally $[6,23-29]$. Results obtained in large detectors under very clean conditions (selected tracks, no background, etc.) are in reasonable agreement with predicted performance. Nevertheless, there are still persistent discrepancies between the predicted relativistic rise including density effect corrections [30] and results of measurements which show systematically smaller values [26.29,31]. Also, the predicted increase of the relativistic rise with reduced sample size $[6,24,26,32]$ was not confirmed experimentally. This subject will be discussed in more detail in connection with "longitudinal drift".

The dependence of the shape of the ionization loss distribution on the sample size is generally determined by changing the gas pressure, which inevitably means change of the gas amplification factor. This difficulty could be avoided by using sums of several "simultaneous" particles (in terms of the time response of the detector and electronics) at the same pressure. Single 4 cm sample distributions of 1, 2, 3 and 4 "simultaneous" $70 \mathrm{GeV} / c$ pions in $\mathrm{Ar}+5 \% \mathrm{CH}_{4}$ at $\mathrm{l}$ and 4 atm are shown in fig. 1 [31]. The most probable values are at channel 45 (42), 98 (92), 154 (144) and 214 (194) for 1 , 2, 3 and 4 particles respectively, at 1 (4) atm. The gas gain at 4 atm was reduced by a factor of 4 with respect to the gain at $1 \mathrm{~atm}$, so that the product of ionization loss and gas gain is roughly the same in both cases. If normalized to the peak value for single particles, the double particle peak is at 2.18 (2.19), for triple particle the ratio is $3.42(3.43)$, the quadruple particle shows onset of slight saturation at higher pressure (ratio 4.76 at 1 atm but only 4.62 at 4 atm). This is in agreement with a factor of about 2.15 for a twofold increase in the sample size, which could be derived from the formula for the most probable ionization loss and which is caused by the logarithmic term containing the sample thickness.

Fig. 2. shows the truncated mean distributions of lowest $40 \%$ values of 64 samples of $4 \mathrm{~cm}$ at 1 and $4 \mathrm{~atm}$ $[31,33]$ corresponding to single sample distributions from fig. 1. Now the peak positions normalized to the single particle distributions are, at 1 and $4 \mathrm{~atm}$, about 2.3, 3.6 and 5.0 for double, triple and quadruple particles respectively. This effect is caused by the sensitivity of the truncated mean to the changes in shape of the ionization loss distribution. It is more evident in fig. 3 [33]

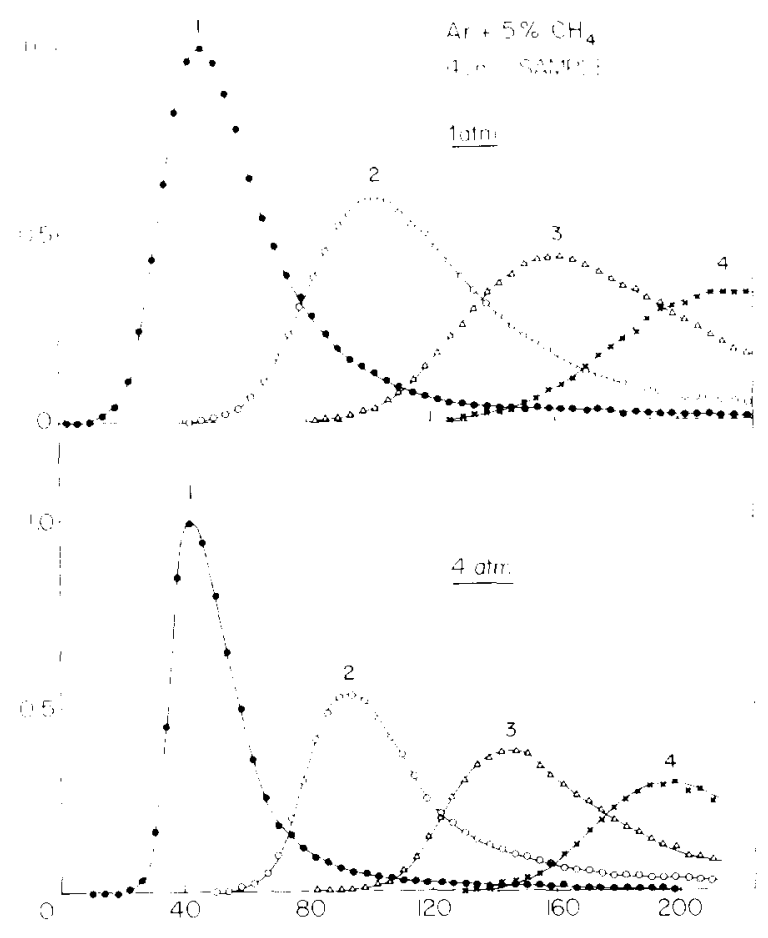

Fig. 1. Single $4 \mathrm{~cm}$ sample pulse height distributions for $1,2,3$ and 4 particles in 1 and 4 atm of $\mathrm{Ar}+5 \% \mathrm{CH}_{4}$

where the retention percentage for the truncated mean is taken as a parameter.

Practically it means that the relativistic rise measured using the truncated mean method for processing of data from this kind of detector will be somewhat improved if a lower percentage is used $(100 \%$ retention corresponds to overall mean and shows obviously a linear behaviour). This effect should be taken into consideration when comparing data obtained under different retention percentages. Lower percentages are clearly advantageous, but there is evidently a practical limit imposed already at about $20 \%$ by greater statistical fluctuations and by increased sensitivity to noise. The final resolution attainable by the truncated mean method is rather insensitive to the choice of the retention percentage in the range from 40 to $70 \%$ [31], but the above mentioned arguments are in favour of the lower percentages close to $40 \%$. It should be noted that in all considerations concerning the $\mathrm{d} E / \mathrm{d} x$ resolution we assume that the detector output signal (including electronics) is really proportional to the energy loss deposit. This means especially that there are no problems with saturation, space charge and dynamic range. It is generally accepted that the gas gain should be kept below $10^{4}$ to avoid difficulties connected to angle dependent corrections for saturation. 

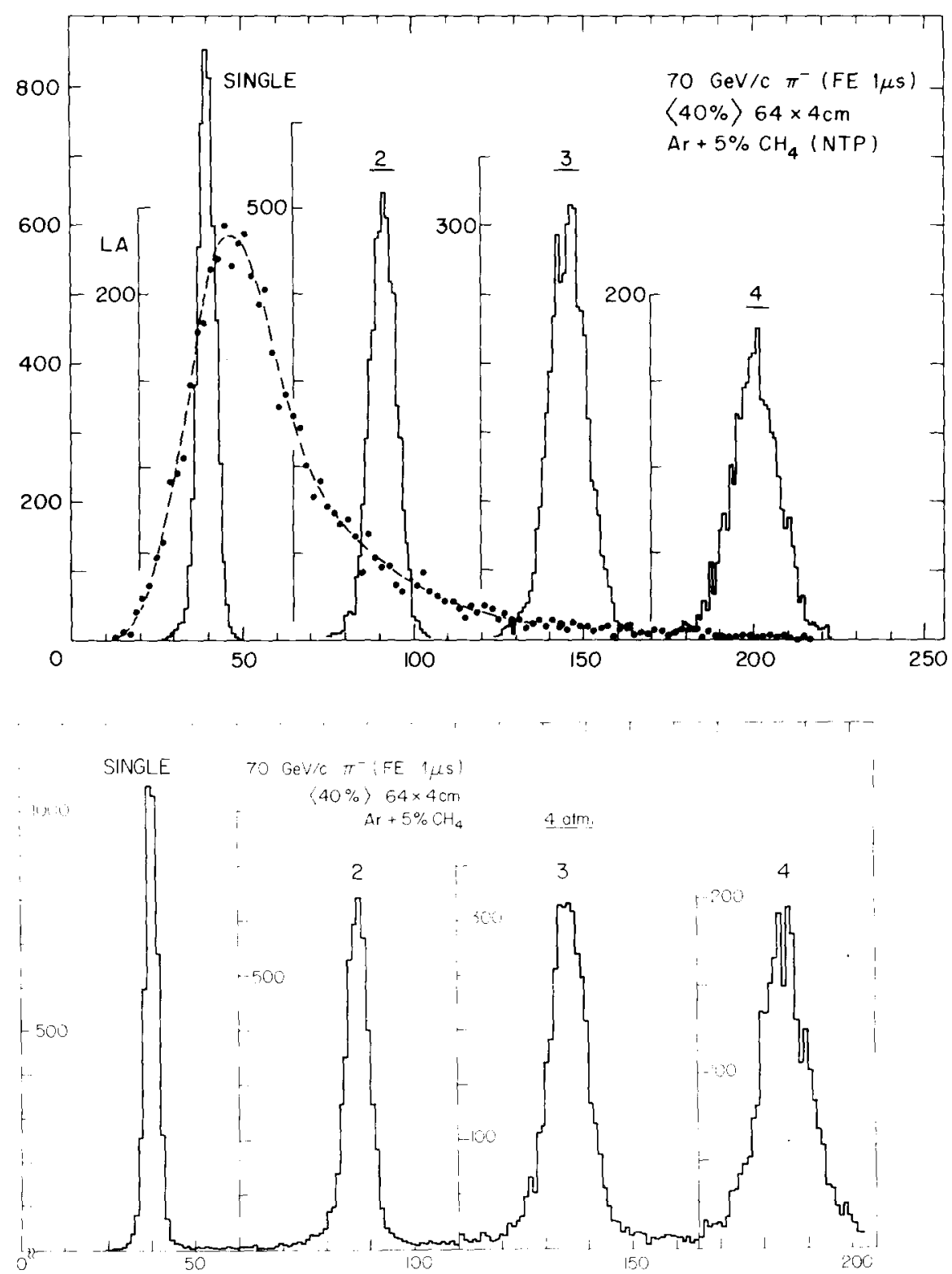

Fig. 2. Truncated mean distributions of $64 \times 4 \mathrm{~cm}$ samples for single, double, triple and quadruple particles. $70 \mathrm{GeV} / \mathrm{c}$ pions in $1 \mathrm{~atm}$ (top) and 4 atm (bottom) of $\mathrm{Ar}+5 \% \mathrm{CH}_{4}$.

\section{Choice of the gas mixture}

In order to attain the best particle identification in a given depth of the detector, we need a gas which has narrow distribution of ionization loss, high relativistic rise and low electron attachment (sensitivity to impurities), especially if the detector contains a large drift space. This will also influence the choice and percentage of the quenching agent, with additional demands for saturated drift velocity and small diffusion dictated by the track reconstruction requirements. In big volume detectors, the choice of gas is also influenced by the economic aspects (cost of gas, open or closed gas system with purification). Concentrating first on the resolution aspects only, we see from fig. 4 [34] where the relative width of the ionization loss distribution of $15 \mathrm{GeV} / c$ pions in a $4 \mathrm{~cm}$ sample at $1 \mathrm{~atm}$ is plotted as a function of the average molecular weight, that heavy hydrocarbons are considerably better than noble gases. Surprisingly, for neon, argon, krypton and xenon the performance is about the same in spite of large differences in density and in the ionization potentials (21.6 


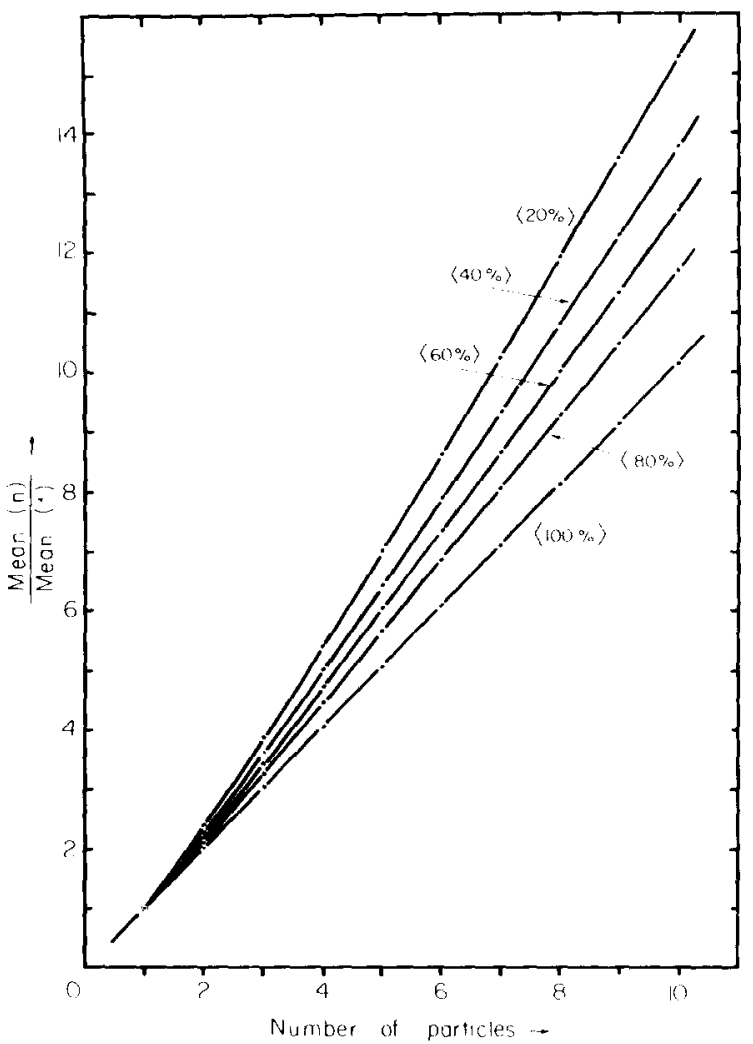

Fig. 3. Truncated mean response for multiple particles with retention percentage as a parameter.

$\mathrm{eV}$ for $\mathrm{Ne} ; 12.1 \mathrm{eV}$ for $\mathrm{Xe}$ ). To compare the particle identification efficiency in various gas mixtures, we need to incorporate the corresponding relativistic rise

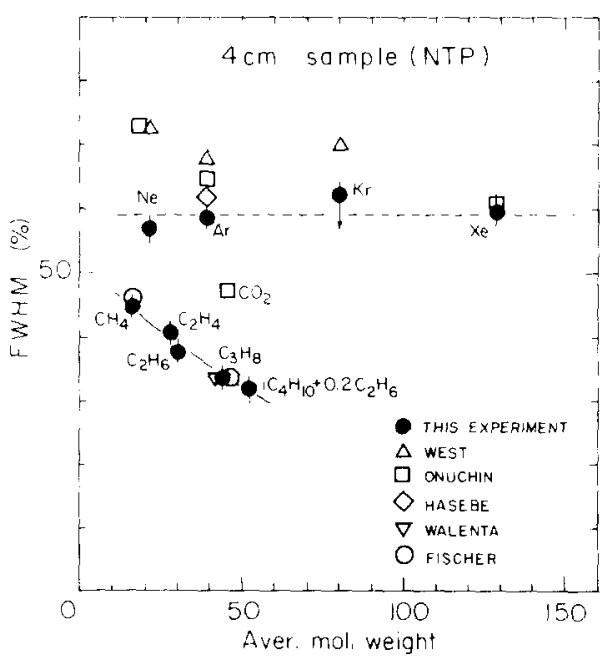

Fig. 4. Single $4 \mathrm{~cm}$ sample resolution as a function of average molecular weight.

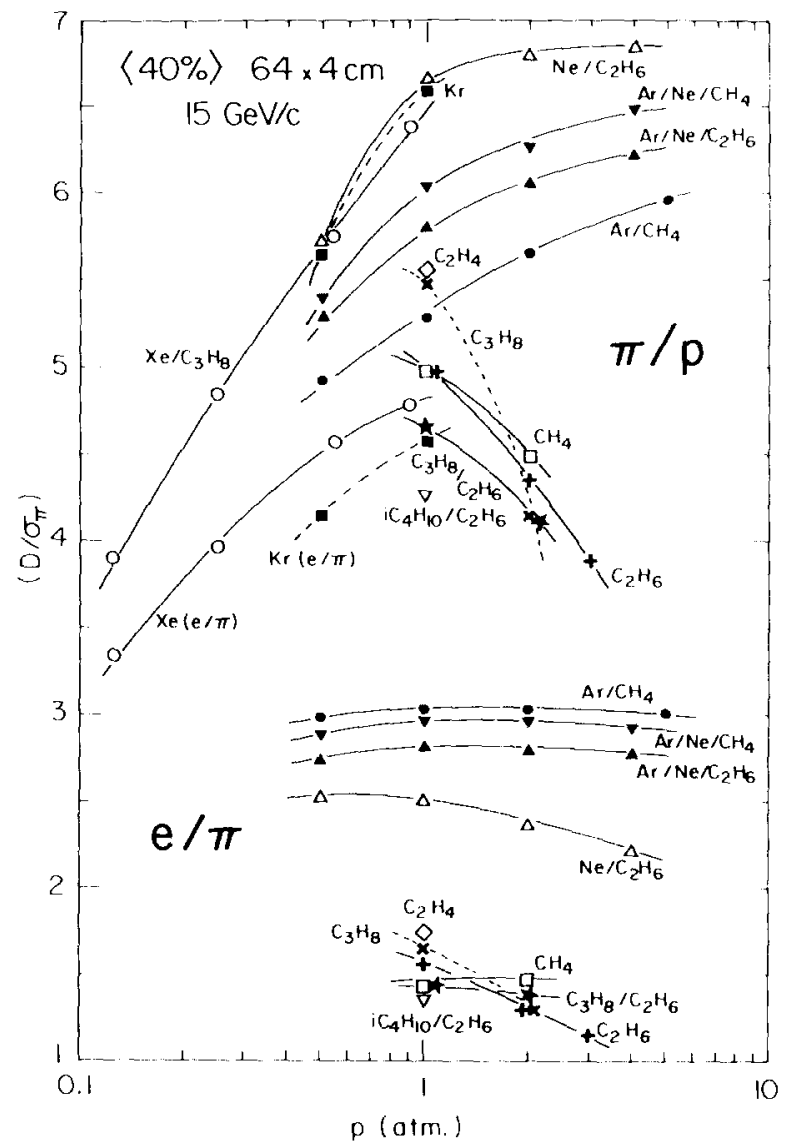

Fig. 5. Identification efficiency at $15 \mathrm{GeV} / \mathrm{c}$ for $\pi / \mathrm{p}$ and $\mathrm{e} / \pi$ for various gases as a function of pressure

for each gas. Fig. 5 [34] shows the resolving power for $\pi / \mathrm{p}$ and $\mathrm{e} / \pi$ at $15 \mathrm{GeV} / \mathrm{c}$, where the distance between various particle peaks is expressed in number of standard deviations as a function of the gas pressure. Truncated mean of $40 \%$ smallest values was used for data from a detector containing 64 samples of $4 \mathrm{~cm}$. At 1 atm neon, krypton and xenon have practically the same merits for $\pi / p$ separation, followed by neon/argon mixtures and by argon. For the interpretation of the gain in the resolving power with increased pressure it should be remembered that this dependence will be different at different particle momenta $(15 \mathrm{GeV} / c$ in fig. 5). Pure hydrocarbons are at atmospheric pressure comparable to argon; the advantage of narrow distributions is offset by small relativistic rise. This is clearly visible in the $\mathrm{e} / \pi$ data where the obtained separation is too small to be of any practical use. Comparison of $e / \pi$ and $\pi / \mathrm{p}$ data for neon and argon indicate steeper slope of the relativistic rise in neon but higher plateau level in argon, so that argon is more efficient for $\mathrm{e} / \pi$ separation. Xenon has the highest relativistic rise, thereby giving the best $\mathrm{e} / \pi$ separation. 


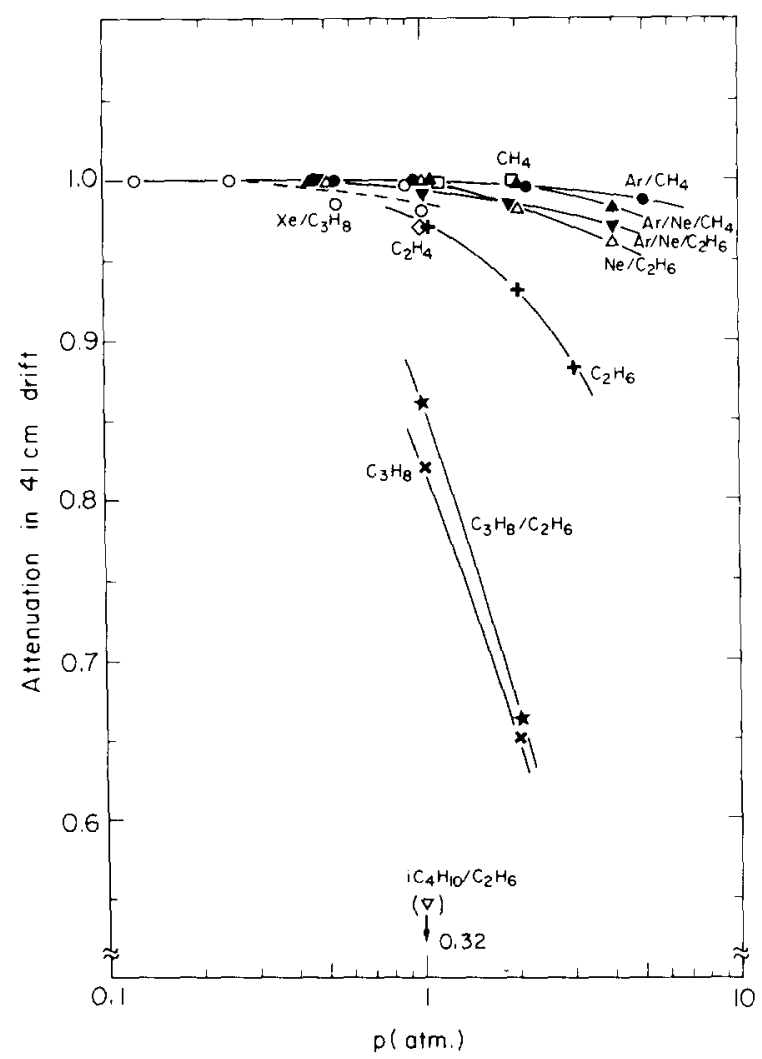

Fig. 6. Signal attenuation over $41 \mathrm{~cm}$ of drift distance for various gases as a function of pressure.

In case of a long drift path in the detector the influence of the attenuation of the ionization signal by attachment must be taken into account. In fig. 6 [34] the level of signal after $41 \mathrm{~cm}$ of drift is plotted as a function of pressure for $\mathrm{Ar} / \mathrm{CH}_{4}, \mathrm{Ar} / \mathrm{Ne} / \mathrm{CH}_{4}$, $\mathrm{Ne} / \mathrm{C}_{2} \mathrm{H}_{6}, \mathrm{Xe} / \mathrm{C}_{3} \mathrm{H}_{8}, \mathrm{CH}_{4}, \mathrm{C}_{2} \mathrm{H}_{4}, \mathrm{C}_{2} \mathrm{H}_{6}, \mathrm{C}_{3} \mathrm{H}_{8}$ and $\mathrm{iC}_{4} \mathrm{H}_{10}$. The gas purity was in the 5-10 ppm $\mathrm{O}_{2}$ range for noble gases and about $15-20 \mathrm{ppm} \mathrm{O}_{2}$ for the hydrocarbons. Argon, neon and methane are remarkably insensitive to attachment, pure hydrocarbons may be acceptable in special cases, for relatively short drift distances. Unfortunately, heavy hydrocarbons are detrimental to the lifetime of the detector (deposit on the electrodes). Results of similar measurements concerning various quenchers in argon [31] show problems with $\mathrm{CO}_{2}$ as a quenching agent. The rate of electron attachment is obviously dependent on the drift velocity, i.e. on the $E / p$ value used.

Drift velocity measurements for a wide variety of gas mixtures are available (see e.g. refs. 29 and 34 and refs. given therein). Certain neon mixtures show saturated drift velocities already at low $E / p$ values which is making them attractive for applications in imaging detectors, if the saturated level of about $3.5 \mathrm{~cm} / \mu \mathrm{s}$ is

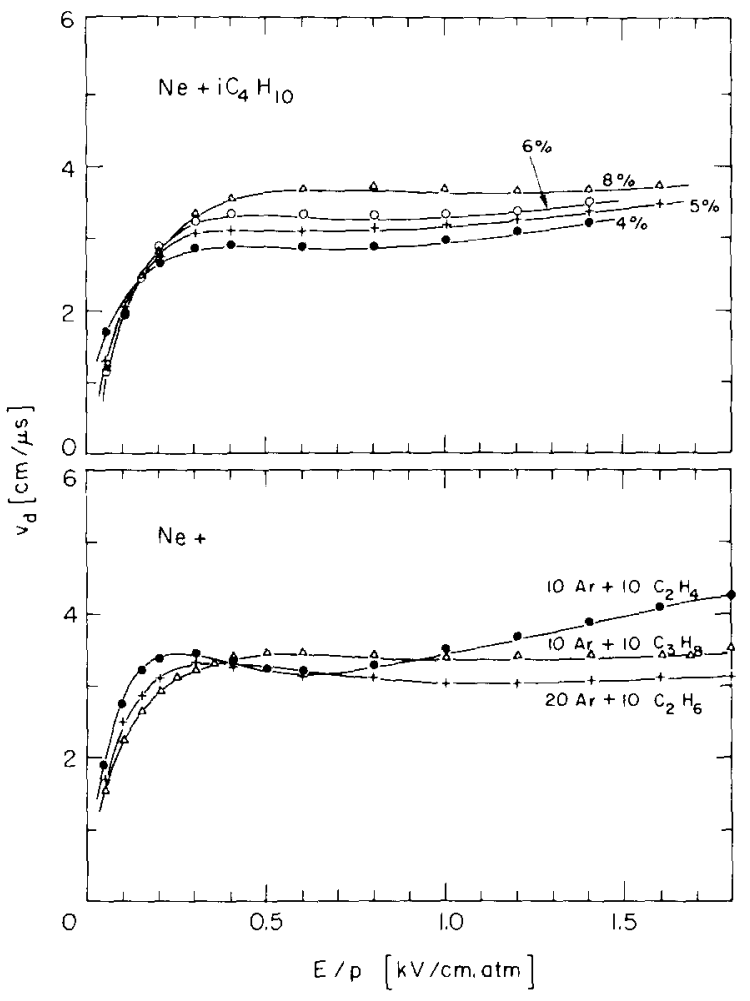

Fig. 7. Drift velocities in mixtures of $\mathrm{Ne} / \mathrm{iC}_{4} \mathrm{H}_{10}$ and in 3-component mixtures of $\mathrm{Ne} / \mathrm{Ar} / \mathrm{C}_{n} \mathrm{H}_{m}$.

acceptable. Examples of drift velocities in $\mathrm{Ne} / \mathrm{iC}_{4} \mathrm{H}_{10}$ and $\mathrm{Ne} / \mathrm{Ar} /\left(\mathrm{C}_{2} \mathrm{H}_{4}, \mathrm{C}_{2} \mathrm{H}_{6}, \mathrm{C}_{3} \mathrm{H}_{8}\right)$ are shown in fig. 7 [29].

The better performance of neon with respect to argon in 64 samples of $4 \mathrm{~cm}$ was also found for $128 \times 2$ $\mathrm{cm}$ samples [29]. The resolution was improved by better control of systematic effects (working without drift) which allowed for gain of one standard deviation in the resolving power. The relativistic rise in neon was measured at several pressures; examples for 1 and 2 atm are shown in fig. 8 together with data for argon from [31]. The higher plateau level in argon and steeper slope in neon were confirmed.

\section{Performance required for relativistic $\pi / K$ separation}

Neglecting for the moment possible conflicts with demands for optimized track reconstruction performance (geometry, gas gain, sample size, drift distance, response of electronics chain and pulse shaping, etc.) we could try to evaluate a set of minimum requirements which will make a "most compact" detector adequate for $\pi / \mathrm{K}$ identification in the relativistic rise region. To 

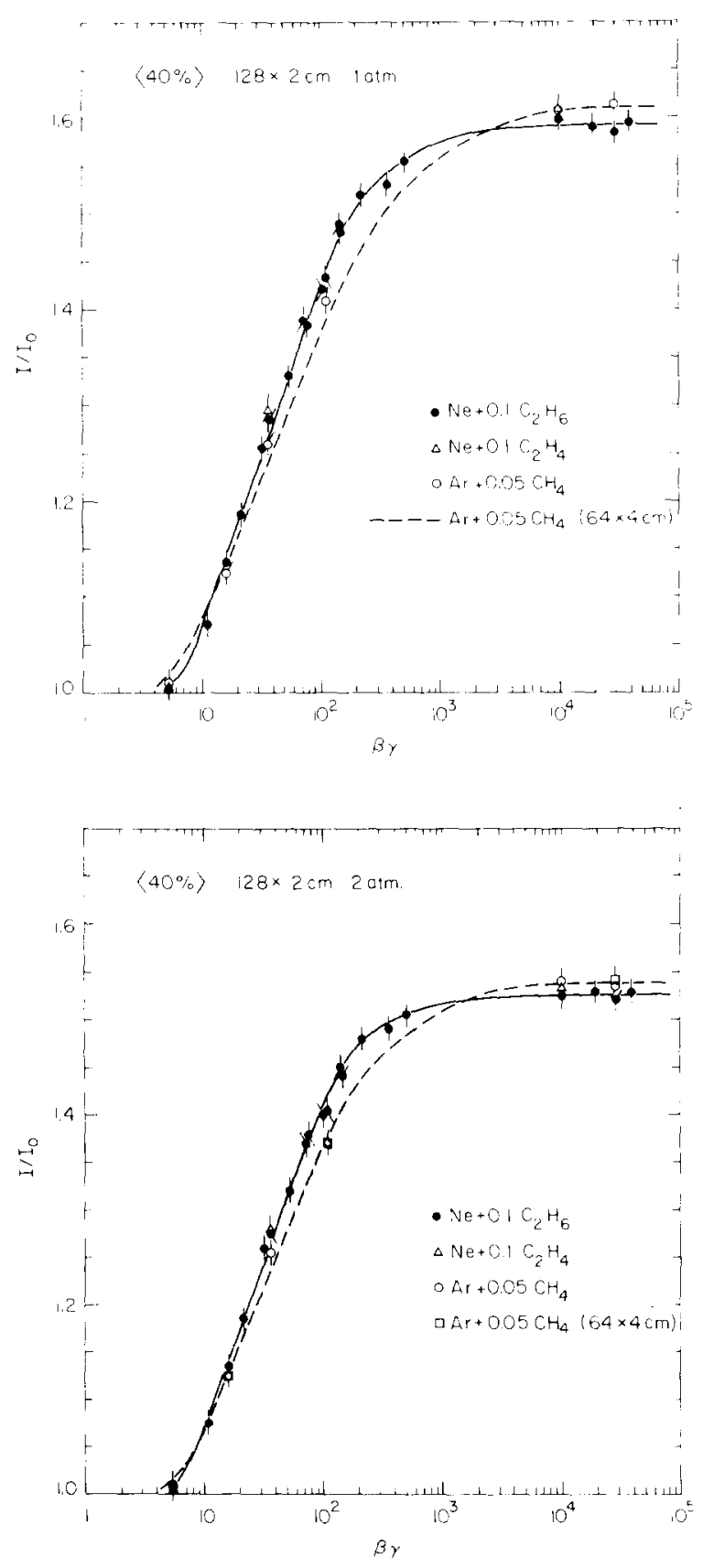

Fig. 8. Relativistic rise at $1 \mathrm{~atm}$ (top) and $2 \mathrm{~atm}$ (bottom) in Ne and Ar.

illustrate the difficulties encountered there, fig. 9 [4] shows the ionization measured on fast forward secondaries from $70 \mathrm{GeV} / c \mathrm{~K}^{+} \mathrm{p}$ interactions. Due to background tracks and related pattern recognition problems causing loss of useful samples, the performance of the detector (EPI, $128 \times 6 \mathrm{~cm}$ samples, $1 \mathrm{~atm} \mathrm{Ar}+5 \%$ $\mathrm{CH}_{4}, 6.6 \%$ fwhm overall resolution attained during

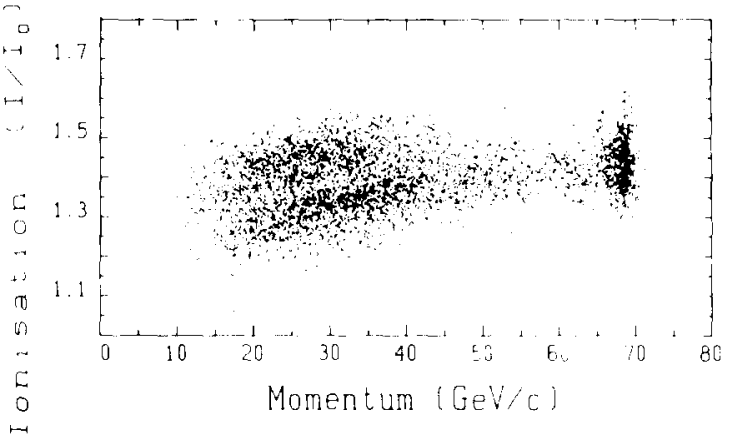

Fig. 9. Ionization measurements for positively charged secondaries from $70 \mathrm{GeV} / \mathrm{c} \mathrm{K}^{+} \mathrm{p}$ interactions in the EPI detector with $>80$ clean samples.

calibration) was reduced to about $8 \%$ fwhm. The bands of pions and kaons are clearly visible, but it will be desirable to have resolution better than the quoted $8 \%$ fwhm $(\sigma / E-3.4 \%)$. In the example of $15 \mathrm{GeV} / c$ protons, pions and electrons measured in $128 \times 2 \mathrm{~cm}$ samples in $\mathrm{Ne} / \mathrm{C}_{2} \mathrm{H}_{6}$ at $2 \mathrm{~atm}$ shown in fig. 10 [29], the width of the distributions is about $7.5 \%$ fwhm.

In large scale devices the final performance is more dependent on the combination of various systematic effects than on the number of samples or sample size, so we could simplify the optimization procedure by concentrating on the required detector depth. A compila-

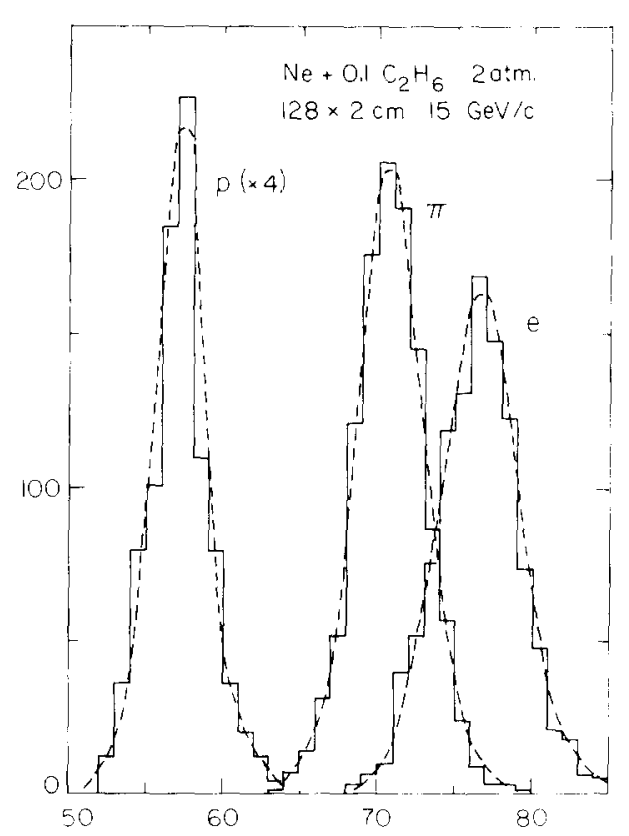

Fig. 10. Truncated mean distribution for $15 \mathrm{GeV} / \mathrm{c}$ protons, pions and electrons in $\mathrm{Ne} / \mathrm{C}_{2} \mathrm{H}_{6}$ at 2 atm. using $128 \times 2 \mathrm{~cm}$ samples. 


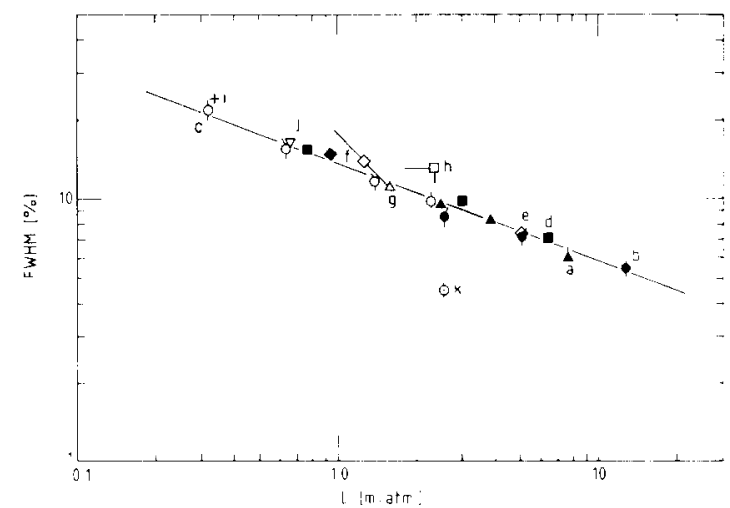

Fig. 11. Resolutions achieved in large detectors as a function of the total detector depth.

tion of resolutions achieved so far in big operational detectors, and in some full scale tests is shown in fig. 11 . The covered range in depth extends above $10 \mathrm{~m} \cdot \mathrm{atm}$ equivalent. The plot in fig. 11 contains data from: (a) EPI, 1/2 EPI [3,4] and EPI test [23]; (b) high pressure results for $\mathrm{Ar} / \mathrm{CH}_{4}$ [29]; (c) low pressure $\mathrm{Xe} / \mathrm{C}_{3} \mathrm{H}_{8}$, assuming rough equivalence with $\mathrm{Ar}$ [34]; (d) LBL TPC [11]; (e) ISIS 1 and ISIS 2 [6,7]; (f) CRISIS test [8]; (g) JADE jet chamber [35]; (h) CLEO $\mathrm{d} E / \mathrm{d} x$ detector [17]; (i) AFS vertex chamber [15]; (j) ARGUS test [36]; (k) pure $\mathrm{C}_{3} \mathrm{H}_{8}$ [29] showing the record performance obtained to date (the arguments against the use of pure hydrocarbons were already discussed). With the exceptions mentioned above, the gas mixture used was in the majority of cases $\mathrm{Ar} / \mathrm{CH}_{4}\left(\mathrm{Ar} / \mathrm{CO}_{2}\right.$ in ISIS and CRISIS, $\mathrm{Ar} / \mathrm{C}_{2} \mathrm{H}_{6}$ in AFS). The plotted results represent always the best performance attained under ideal conditions, i.e. for selected events with limited background. Some of the "error bars" indicate degradation of resolution under severe background conditions. The depth dependence could be expressed with reasonable precision by $13.5 L^{-0.37}$ (drawn on the graph). If the $8 \%$ fwhm limit in resolution is to be attained, depth of at least $4 \mathrm{~m}$-atm will be needed. Estimations based on semi-empirical formulas $[23,26,28]$ give comparable results. The atmospheric pressure version of such a detector will not fit into the space available in colliding beam applications. Increasing the pressure will improve the situation, providing that the inevitable pressure vessel could be tolerated and, furthermore, that the gain in resolution is not of fset by considerable decrease in the relativistic rise. Fortunately, the influence of the pressure on the slope of the relativistic rise is not very strong, so that in the first approximation only the maximum momenta will be affected. An operational pressure near 2 atm is clearly the best for practical reasons. The pressure vessel walls can be kept reasonably thin and the pump-down option offers great ad-

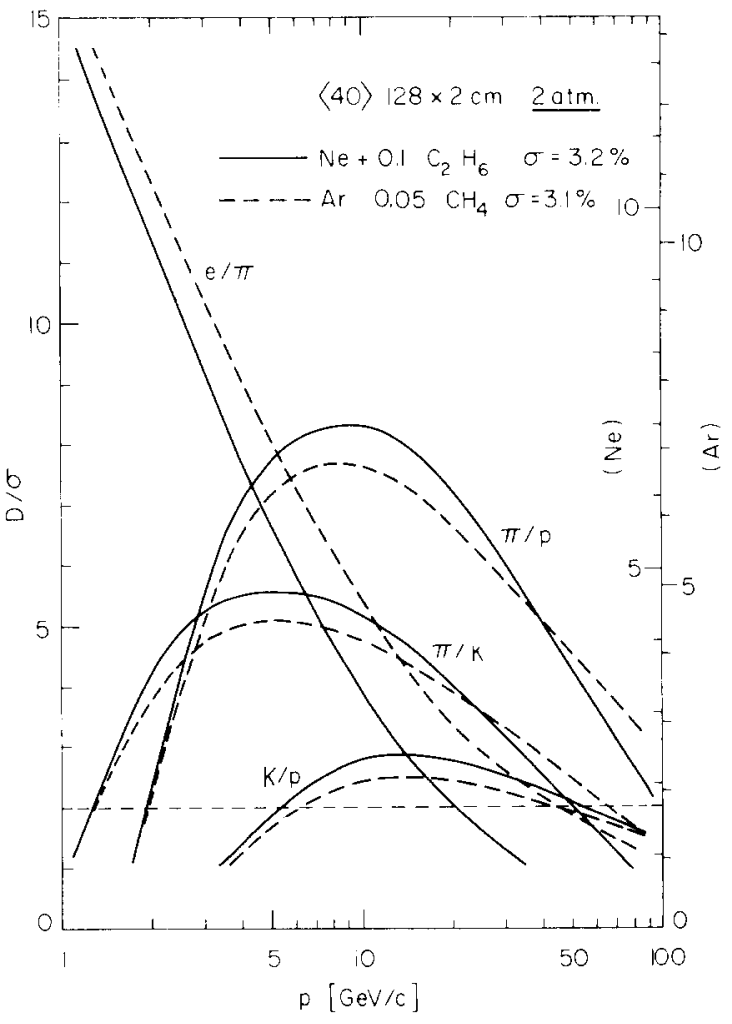

Fig. 12. Expected particle separation in $\mathrm{Ne}$ and $\mathrm{Ar}$ at $2 \mathrm{~atm}$. Truncated mean of $128 \times 2 \mathrm{~cm}$ samples.

vantages in control of the gas purity. Fig. 12 [29] shows as an example the particle identification efficiency for a detector of $5.12 \mathrm{~m} \cdot \mathrm{atm}(2.56 \mathrm{~m}$ at $2 \mathrm{~atm})$ equivalent depth, with resolution close to $7.5 \% \mathrm{fwhm}$. The full curves are for neon and the dashed ones for argon. The sample size is $2 \mathrm{~cm}$, the resolving power $(D / \sigma)$ scale on the right is for a scaled-down version of $128 \times 1 \mathrm{~cm}$ samples at $2 \mathrm{~atm}$. The differences in favour of neon (with the exception of $\mathrm{e} / \pi$ separation) were found to be more pronounced for large sample size, which indicates that $(4 \mathrm{~cm} \cdot \mathrm{atm})$ is already well below the optimum value in neon. For argon the optimum sample size is about $5 \mathrm{~cm} \cdot \mathrm{atm}$. In our previously proposed limit case of $4 \mathrm{~m} \cdot$ atm detector depth, we could expect the $\pi / \mathrm{K}$ separation at a level of $\geqslant 4 \sigma$ from -3 to $\geqslant 20 \mathrm{GeV} / c$. Use of neon will allow for operation at lower pressure, if a certain loss in the e/ $\pi$ separation efficiency with respect to argon is acceptable.

It is difficult to discuss in sufficient detail all the aspects of the influence of the electronics design (ac coupling, baseline shifts, pulse shaping response, pile-up, etc.) on the detector performance. We shall concentrate only on problems related to the shape of the ionization loss distributions with respect to the available dynamic range of the electronics chain. This is especially im- 


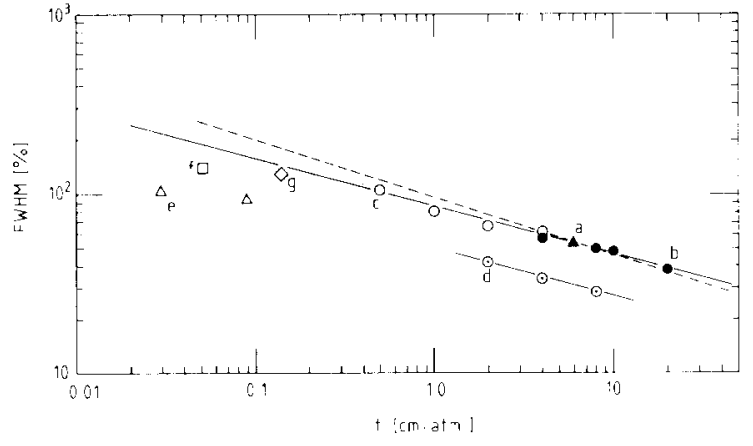

Fig. 13. Relative width of single sample ionization loss distributions as function of sample size.

portant for relatively small samples below $1 \mathrm{~cm} \cdot \mathrm{atm}$ where only a limited number of reliable distribution measurements is available. Those measurements were realized at reduced pressure in samples of depth of several $\mathrm{cm}$. The dependence of the width of single sample ionization loss distribution on the sample size is shown in fig. 13. The data in the plot are from: (a) EPI [3,4] for $\mathrm{Ar} / \mathrm{CH}_{4}$ at $1 \mathrm{~atm}$; (b) high pressure result in $\mathrm{Ar} / \mathrm{CH}_{4}$ [29]; (c) low pressure in $\mathrm{Xe} / \mathrm{C}_{3} \mathrm{H}_{8}$, assuming similar behaviour for all noble gases as illustrated in fig. 4 [34]; (d) pure $\mathrm{C}_{3} \mathrm{H}_{8}$ results for comparison [29,34]. The distribution width follows reasonably well a $\left(85 t^{-0.26}\right)$ dependence (full line in the graph). The discrepancy with the $\left(96 t^{-0.32}\right)$ dependence proposed in ref. 27 (dashed line) is within acceptable limits. Results for argon from ref. 26 are comparable. The distributions measured at low pressures could therefore be used for sample depth $\leqslant 0.5 \mathrm{~cm} \cdot \mathrm{atm}$. Fig. 14 shows examples of such distributions, derived from [34]. It corresponds to about $0.4 \div 0.5 \mathrm{~cm}$ of argon at $1 \mathrm{~atm}$. The first distribution is for a particle at the minimum of ionization ( $\sim 40$ ion pairs, $\sim 600 \mathrm{eV}$ energy loss). The second distribution is for the plateau of the relativistic rise $\left(\sim 1.6 I_{0}\right)$. The relative width is about $130 \%$ in the first and $-115 \%$ fwhm in the second case. The arrows on the top indicate shifts of the peaks which will be typically caused by $\pm 20 \mathrm{mb}$ (maximum encountered) variations in the atmospheric pressure. Introducing realistic angular corrections for track length in both planes with respect to the signal wire will produce (with some reserve) the last distribution at about $4 I_{0}$. Note that the plotted distributions are not normalised. The position of the $I_{0}$ peak could be shifted towards the low values, if the signal to noise ratio will remain at acceptable level. This is important, because the truncated mean method "prefers" the low ionization values. At the upper end we see the limit of the dynamic range of the 8-bit linear ADC. Fig. 15 shows the distributions from fig. 14 "processed" in an ADC with logarithmic response. The dashed distribution represents the mentioned reserve if the noise level is tolerable. A linear 9-bit ADC will have adequate dynamic range. In principle, the dynamic range of a "logarithmic" ADC could be expanded or compressed within certain limits [37], but it seems that 6-bit $\log$ ADC will be just sufficient. If such wide dynamic range will be not needed, the actual ADC resolution of

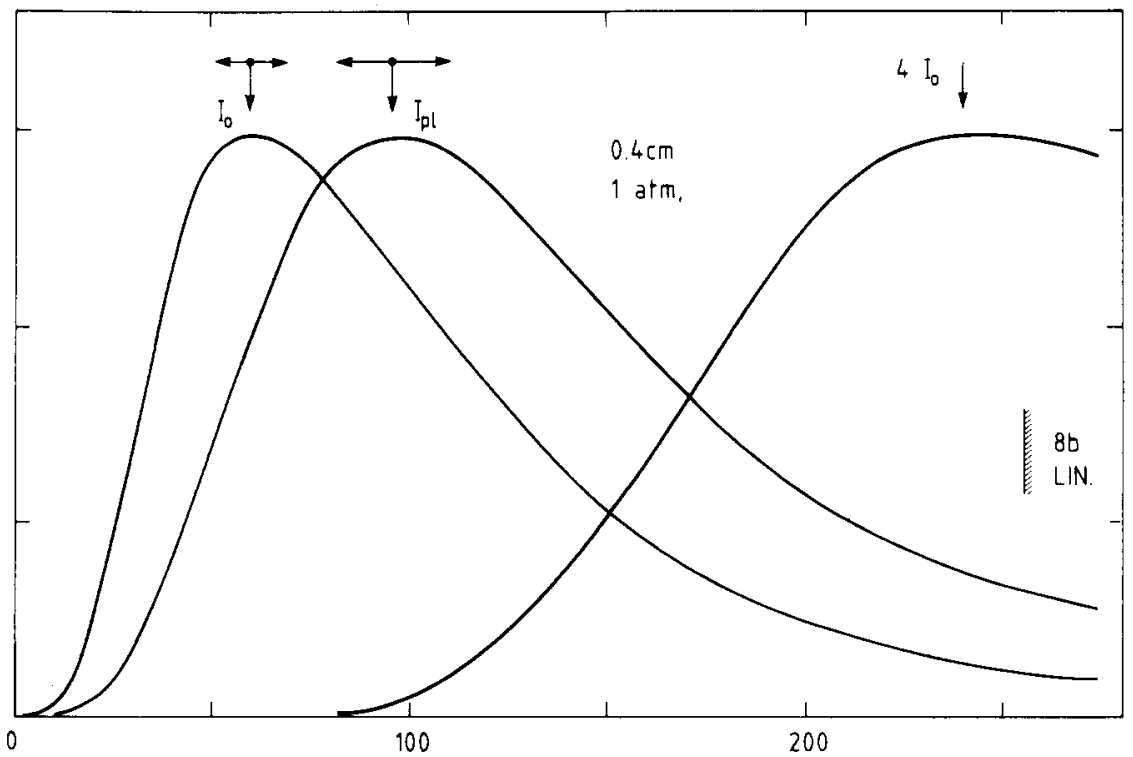

Fig. 14. Examples of single $0.4 \mathrm{~cm}$ sample distributions at $1 \mathrm{~atm}$. Particles at minimum ionization, on the plateau, and with maximum correction for angular effects. 


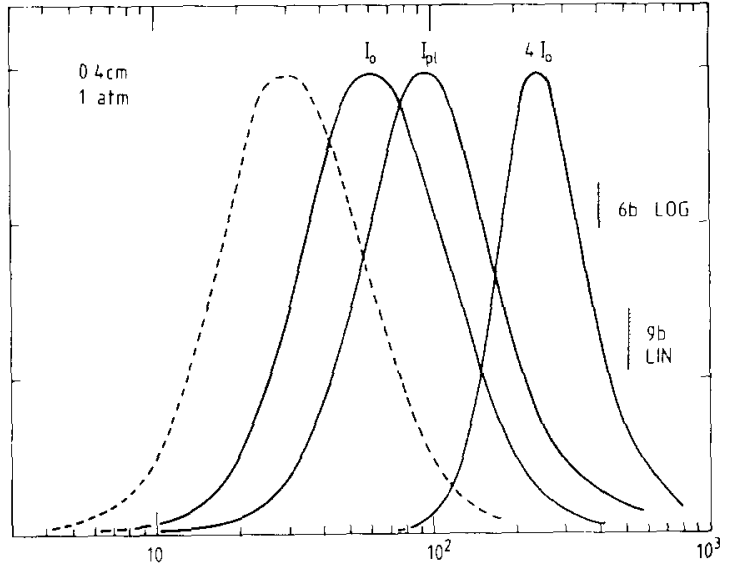

Fig. 15. Distributions from fig. 14 processed in a logarithmic response $\mathrm{ADC}$.

only 4 bits will be sufficient (the errors are dominated by the fluctuations in the ionization loss).

\section{Longitudinal drift and fine sampling}

As already mentioned in sect. 2, a potential improvement in the efficiency of the particle identification will be in using many very fine sampling intervals [38]. Recently, several experiments were realized in order to investigate the performance of detectors where the ioni- zation deposited in the detector drifted along the particle trajectory and the fine sampling was obtained by flash ADCs at $10-40$ ns intervals. With fast shaping amplifier response and at moderate drift velocity the corresponding sample "size" was below $1 \mathrm{~mm}$. Improvements of resolving power by about a factor of two were quoted from prototype measurements $[39,40]$, when selected part of the data was extrapolated to $1 \mathrm{~m}$ of total track length.

To illustrate the method fig. 16 shows (top) an example of a single $15 \mathrm{GeV} / c$ pion detected in 1 stage (4.7 $\mathrm{cm}$ of drift) of a 16-stage detector [41] in $\mathrm{Ar} / \mathrm{CO}_{2}$ and with 25 ns sampling interval, corresponding to about $0.3 \mathrm{~mm}$ sample "size". Most of the large individual ionization clusters are visible, the small clusters were smeared by diffusion and not resolved. The bottom part of fig. 16 depicts the result of accumulation of many such events under the same conditions. The peak at the beginning is caused by the addition of ionization from both sides of the signal wire inside the proportional cell. Due to limitations imposed by the response of the shaping circuits, only 133 samples per stage ("flat part" in fig. 16) could be used in this case, so that from the total detector length of $88 \mathrm{~cm}$ only $64 \mathrm{~cm}$ were "active" for particle identification. A similar example is shown for $\mathrm{Ne} / \mathrm{C}_{3} \mathrm{H}_{8}$ in fig. 17 (top). The sample "size" corresponding to $25 \mathrm{~ns}$ sampling rate was about $0.9 \mathrm{~mm}, 35$ samples per stage were used. In the bottom part of fig. 17 are shown the final distributions of a truncated mean of $40 \%$ smallest values for $15 \mathrm{GeV} / c$ protons, pions and
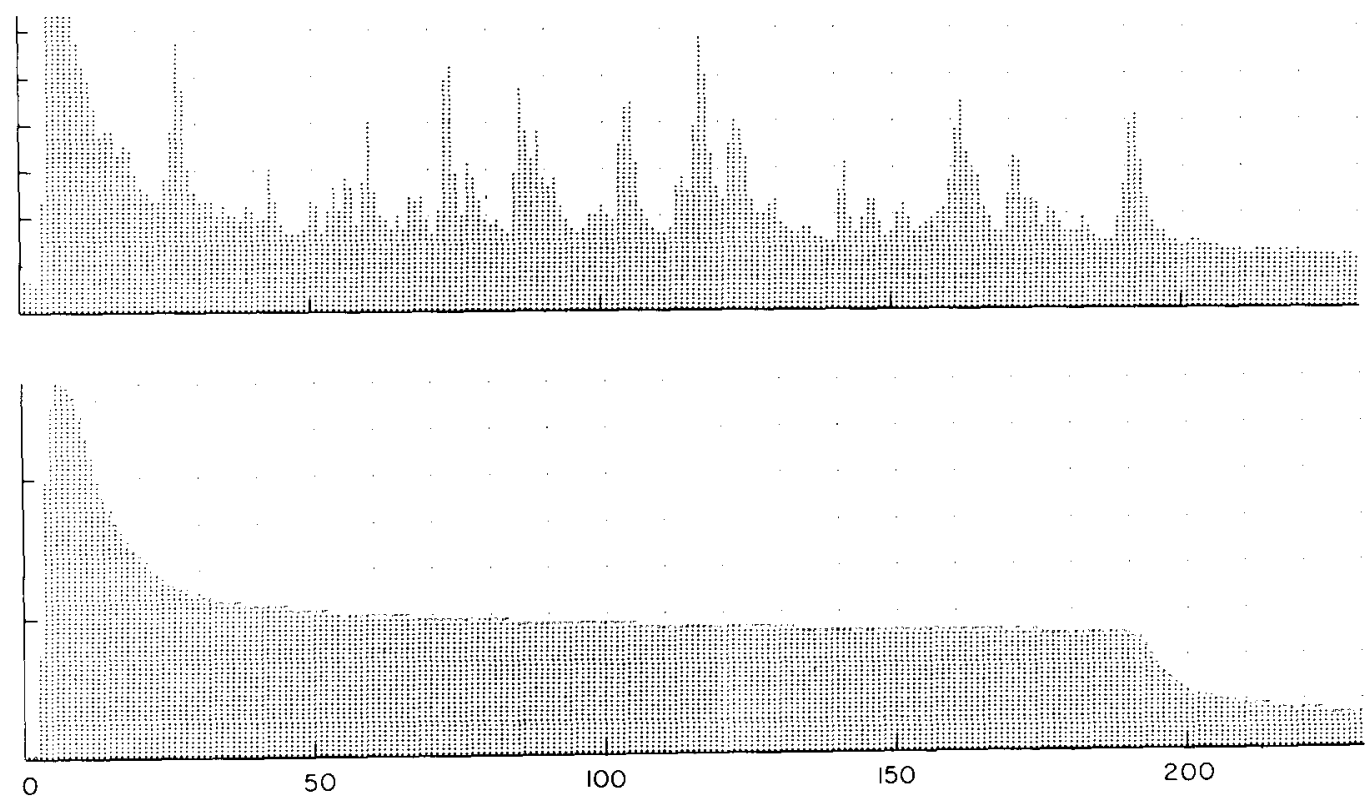

Fig. 16. Example of a flash ADC response over $4.7 \mathrm{~cm}$ of longitudinal drift ( $25 \mathrm{~ns} / \mathrm{bin}$ ) for one $15 \mathrm{GeV} / \mathrm{c}$ pion passing through one stage of the detector filled with $\mathrm{Ar} / \mathrm{CO}_{2}$ at 1 atm (top). Summed response for many particles in all 16 stages of the detector (bottom). 


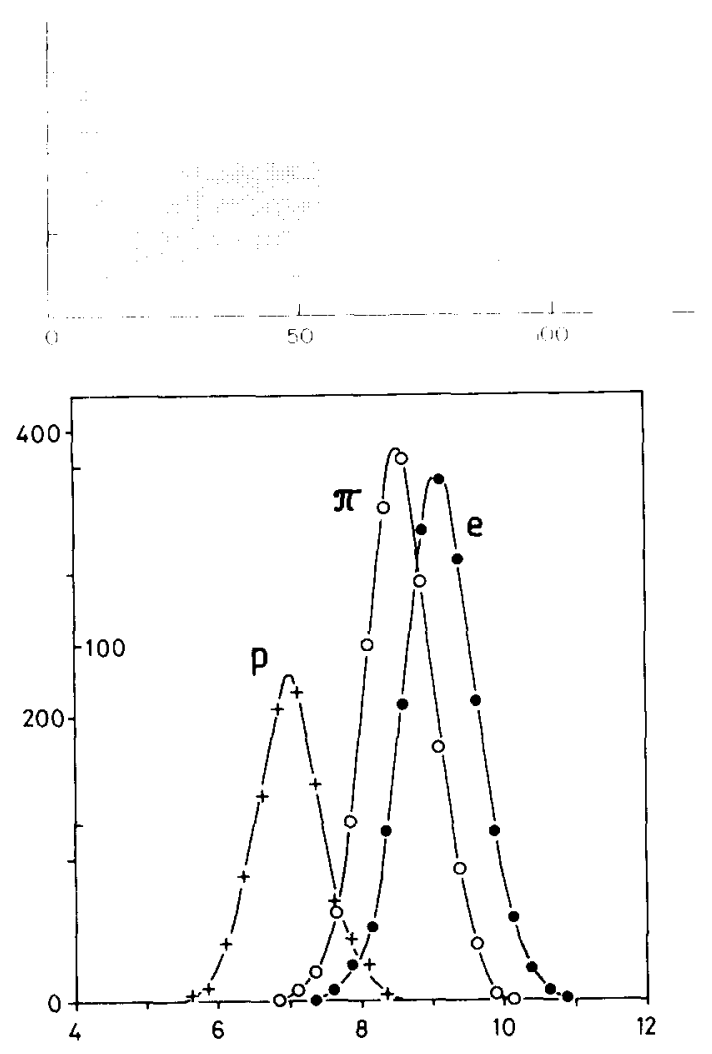

Fig. 17. The FADC response (25 ns/bin) summed over 16 stages and many particles for $\mathrm{Ne} / \mathrm{C}_{3} \mathrm{H}_{\mathrm{x}}$ (top) and distributions of truncated means ( $40 \%$ smallest values) for protons, pions and positrons at $15 \mathrm{GeV} / \mathrm{c}$ in $\mathrm{Ne} / \mathrm{C}_{3} \mathrm{H}_{8}$, using 560 samples per track (bottom).

electrons, in $\mathrm{Ne} / \mathrm{C}_{3} \mathrm{H}_{8}$. The resolution for pions is in the range of $15 \div 20 \% \mathrm{fwhm}$, which is consistent with the predicted value for about $50 \mathrm{~cm}$ detector length in fig. 11. Also, the $e / \pi / p$ ratios are comparable to results obtained by classical charge integration over large samples [29.34]. Similar behaviour has been found for several different gas mixtures for a variety of drift velocities. So it seems that apart from technical difficulties which will arise in construction of large volume detectors of this type, there is virtually no improvement in performance which will allow for reduction of the detector size. Measured widths of the single "fine" sample distributions were added for comparison into fig. 13: (e) $\mathrm{Ar} / \mathrm{CO}_{2}$ $(0.3 \mathrm{~mm})$ and $\mathrm{Ar} / \mathrm{C}_{3} \mathrm{H}_{8}(0.9 \mathrm{~mm})$ [41]; (f) $\mathrm{Ar} / \mathrm{CO}_{2}[40]$; (g) $\mathrm{Ar} / \mathrm{CH}_{4}$ [39]. Compared to values expected by extrapolation from "large" samples, the "fine" samples distributions appear narrower. This may be an indication that the content of "fine" sample is not correctly defined by the drift distance between two FADC samples. Indeed, the average distance between the primary ionization clusters, electron diffusion during the drift, sampling interval and the shaping time constants are of the same order, which may introduce correlation effects.

\section{Amplitude calibration using laser beams}

Recently, use of laser induced ionization for simulation of charged particle tracks was introduced and is now widely used $[42-49]$. In spite of the fact that the exact mechanism of (double) excitation is not yet fully understood, pulsed UV lasers are currently used for generation of long straight tracks in big detectors, for position calibration, drift velocity and diffusion measurements, measurements of space charge effects, etc. Compared to pulsed X-ray sources used previously [19]. present two-stage $\mathrm{N}_{2}$ lasers are compact and easy to operate. The required UV transparent windows could be incorporated into the detector without great difficulties. Another attractive features of the use of laser beams is the possibility of beam splitting for studies of two-track resolution. We will concentrate here on the applications aimed at amplitude measurements $(=\mathrm{d} E / \mathrm{d} x$ ) over long tracks, without any photo-ionizing additives in the gas.

Using pulse length of $-300 \mathrm{ps}$ fwhm, energy density $\geq 10 \mu \mathrm{J} / \mathrm{mm}^{2}$ at $337 \mathrm{~nm}$ (photon energy $3.7 \mathrm{eV}$ ) is required to produce -100 electrons $/ \mathrm{cm}$ in $\mathrm{Ar}$ at $1 \mathrm{~atm}$, which corresponds to the energy loss of relativistic particles. The number of electrons was found to depend quadratically on the energy density up to about 20 $\mu \mathrm{J} / \mathrm{mm}^{2}$, with a transition to linear dependence at higher energy densities. This transition was reproduced in several experiments, which is somewhat surprising, because it indicates presence of a common photo-ionizing impurity in similar concentrations (the $3.7 \mathrm{eV}$ photons from the $\mathrm{N}_{2}$ laser cannot ionize directly the argon

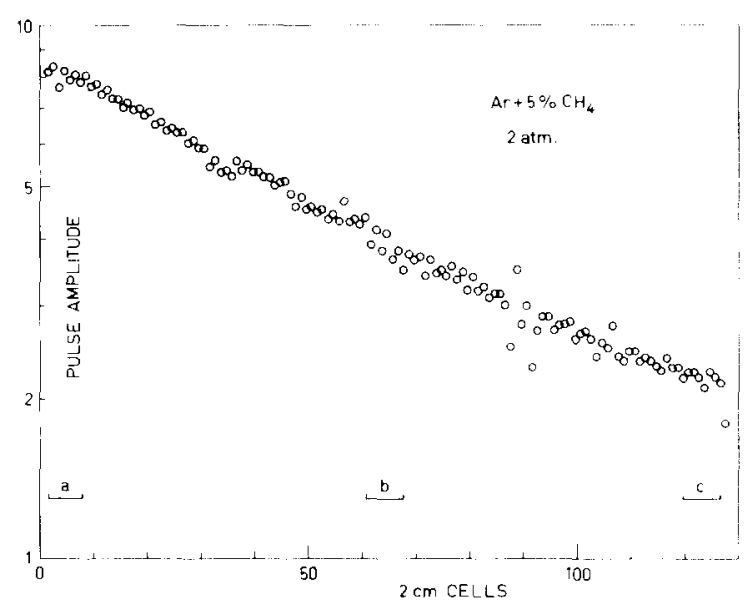

Fig. 18. Pulse amplitudes measured simultaneously using $\mathrm{N}_{2}$ laser tracks through $2.56 \mathrm{~m}$ long detector. Beam enters at cell 0 . Uncorrected data in $\mathrm{Ar} / \mathrm{CH}_{4}$ at 2 atm, no focusing. 


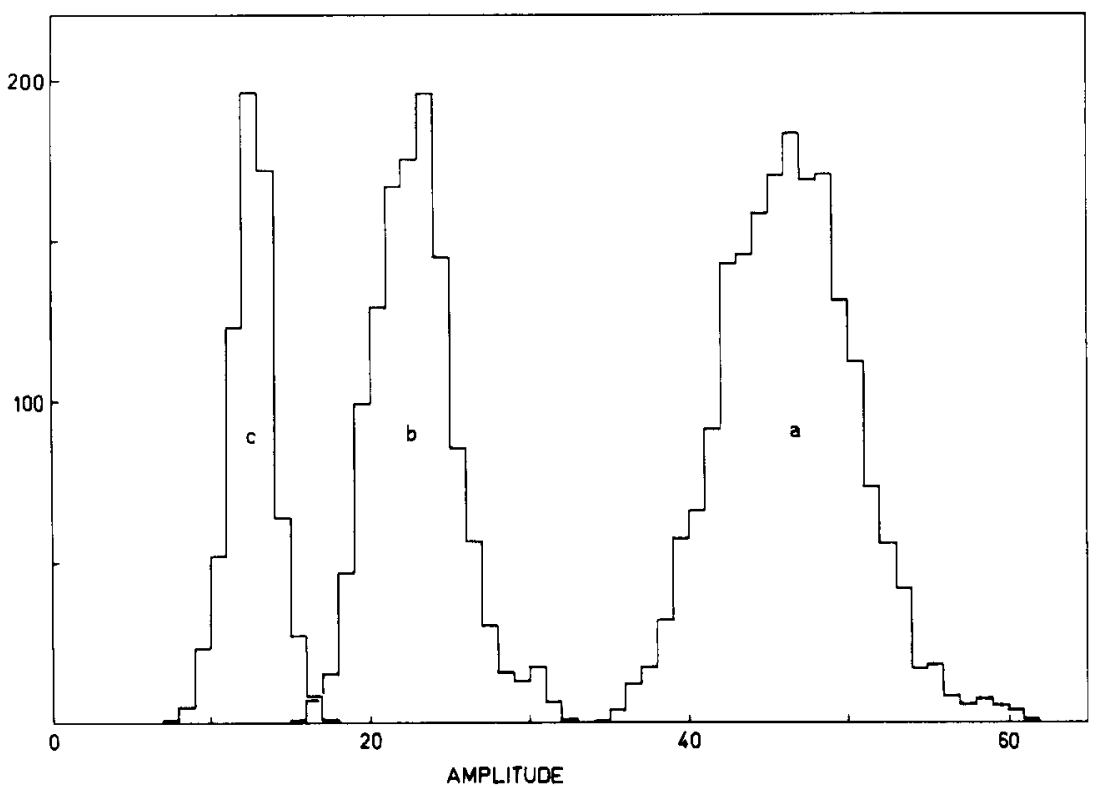

Fig. 19. Amplitude distributions from groups of adjacent cells from fig. 18 at the detector entry (a), near centre (b) and at the exit (c). Laser intensity fluctuations ( $14 \%$ fwhm) not removed.

atoms with ionization potential of $15.7 \mathrm{eV}$ ). The dimensions of the laser beam spot could be made sufficiently small by adequate collimation, with beam divergency limited essentially by diffraction (at least in one plane). If the available energy density is high enough to work in the linear range, track lengths above $2 \mathrm{~m}$ could be used for amplitude calibration. Single lens focusing cannot be applied, but the use of an afocal system [48] may produce even more uniform beam over the same distance. Pulse-to-pulse intensity variations could be to great extent corrected for by using a reference photodiode. Almost symmetric pulse height distributions were obtained, with relative width $<20 \%$ fwhm. In a two-track resolution study [49] onset of saturation at gas amplification factor $\sim 5 \times 10^{3}$ has been found, which is consistent with the generally accepted value in argon.

In principle, lasers cannot be used for absolute calibration for $\mathrm{d} E / \mathrm{d} x$ purposes, due to long-term stability problems (beam area, halo, unknown photosensitive impurities in the gas, etc.). Reliable relative calibration (mapping of local non-uniformities) will depend on the level of control over the beam area and energy density. As an example, fig. 18 shows uncorrected data from amplitude measurements realized in our group at CERN. The track length is $2.56 \mathrm{~m}$ in $2 \mathrm{~atm}$ of $\mathrm{Ar} / \mathrm{CH}_{4}$ mixture. A two-stage $300 \mathrm{ps}$ pulse length $\mathrm{N}_{2}$ laser was used, with the halo removed by collimation, without focusing. The laser output was $240 \mu \mathrm{J}$ and the measured pulse amplitude at the detector entry was about 3 times higher than ionization signal from relativistic particle tracks. The fluctuations of the laser intensity (14\% fwhm on a reference photodiode) were not removed. Uncorrected distributions from groups of channels at the detector entry, near the centre and at the exit are shown in fig. 19. The relative widths are 20, 24 and $26 \%$ fwhm respectively. The amplitude ratio from entry to exit is about $4: 1$ and the dependence on distance along the track is roughly exponential (the amplitude scale is arbitrary). More sophisticated (YAG) lasers working at different wavelength and with beam of better quality will make the amplitude measurements more reliable.

\section{Conclusions}

Particle identification by ionization sampling could be easily combined with the trajectory determination in the central vertex chambers of large colliding beam detectors. If a compromise solution to conflicting demands for optimized track reconstruction (diffusion, drift velocity, gas gain) could be found (separate functions?), at least $4 \mathrm{~m} \cdot$ atm equivalent path will be needed for reliable $\pi / \mathrm{K}$ separation in the relativistic rise region. $\mathrm{Ar} / \mathrm{CH}_{4}$ is the best gas mixture for application in large drift volumes, because of its low sensitivity to impurities. Use of neon will allow for reduction in the gas pressure with respect to argon. $\mathrm{K} / \mathrm{p}$ separation is marginal in any case, $e / \pi$ separation will be better in argon. 
Optimum $\mathrm{d} E / \mathrm{d} x$ performance extending to higher momenta will be obtained at atmospheric pressure. Use of (moderate) pressure will open the attractive option of pump-down of the pressure vessel (less problems with the gas purity).

The $\mathrm{d} E / \mathrm{d} x$ sampling will be most efficient in a detector geometry with classical (small) drift cells of the ARGUS type, which could work with single hit electronics without problems related to space charge (no gating required). A bicycle wheel geometry similar to the jet chamber one for JADE with moderate drift distances will suffer less from the systematic effects than a TPC detector with much longer drift. There the space charge problems already affect the track reconstruction precision and a perfect gating system is required [50]. The TPC is also very sensitive to impurities in the gas.

In general, the $\mathrm{d} E / \mathrm{d} x$ sampling efficiency will be better if a sample size $>1 \mathrm{~cm} \cdot \mathrm{atm}$ is used. There is no hard experimental evidence for certain predictions of improved performance for smaller sample size.

In order to reach the desired particle identification efficiency, all systematic effects must be fully under control. Detailed analysis of parameters affecting the detector performance is not possible here, so I will simply make a list of the most important ones, together with some potential problems:

Mechanical tolerances and stability (temperature, pressure, electrostatic forces).

- Uniformity of electric and magnetic field in the drift space.

- Absolute amplitude response calibration, control and monitoring (gas pressure and temperature, mixture stability, gas purity, stability of the power supplies and of electronics gain, etc., long-term monitoring precision, especially if operating at atmospheric pressure - see example in ref. 4).

Background problems (space charge, gating efficiency for long drift distance, saturation effects).

- Large corrections for inclined and curved tracks.

- Response of the electronics chain (noise, stability, pulse shaping, baseline shifts, crosstalk, dynamic range, etc.).

Some of the important design features could be easily tested using laser generated tracks; the final performance of a big detector could be assessed only in particle beam tests.

\section{References}

[1] D. Jeanne et al., Nucl. Instr. Meth. 111 (1973) 287.

[2] M. Aderholz et al., Nucl. Instr. and Meth. 123 (1975) 237.

[3] P. Lazeyras et al., IEEE Trans. Nucl. Sci. NS-26 (1979) 89.

[4] V. Baruzzi et al., Nucl. Instr. and Meth. 207 (1983) 339.

[5] W.W.M. Allison et al., Nucl. Instr. and Meth. 119 (1974) 499
[6] W.W.M. Allison, Proc. Int. Conf. on Instrumentation for colliding beam physics. SLAC-250 (1982) p. 61

[7] M. Aguilar-Benitez et al.. Nucl. Instr. and Meth. 205 (1983) 79 .

[8] B.F. Wadsworth et al., IEEE Trans. Nucl. Sci. NS-26 (1979) 120.

[9] PEP-4; PUP-5012 (1976); D. Fancher et al., Nucl. Instr. and Meth. 161 (1979) 383

[10] G.R. Lynch and N.J. Hadley, Proc. Int. Conf. on Instrumentation for colliding beam physics. SLAC-250 (1982) p. 85.

[11] H. Aihara et al.. Proc. 1982 Nuclear Science Symp., IEEE Trans. Nucl. Sci. NS-30 (1983) 63.

[12] J. Heintze, Nucl. Instr. and Meth. 156 (1978) 227: W. Farr et al. Nucl. Instr. and Meth. 156 (1978) 283.

[13] H. Drumm et al., Nucl. Instr. and Meth. 176 (1980) 333; J. Heintze, Proc. INS Int. Symp. on Nuclear radiation detectors. Tokyo (1981). Nucl. Instr. and Meth. 196 (1982) 293.

[14] S. Almehed et al., CERN/ISRC 76-36/P95 (1977).

[15] O. Botner et al., Nucl. Instr. and Meth. 196 (1982) 315.

[16] E. Nordberg and A. Silvermann, CBX 79-6, Cornell Univ.

[17] R.D. Ehrlich, Proc. Int. Conf. on Instrumentation for colliding beam physics, SLAC-250 (1982) p. 71

[18] M. Barranco-Luque et al., Nucl. Instr. and Meth. 176 (1980) 175 .

[19] M. Calvetti et al., IEEE Trans. Nucl. Sci. NS-30 (1983) 71

[20] Phys. Scripta 23 (1981) no. 4.

[21] SLAC-250 (June 1982).

[22] IEEE Trans. Nucl. Sci. NS-30 (1983).

[23] M. Aderholz et al., Nucl. Instr. and Meth. 118 (1974) 419.

[24] V.K. Ermilova et al., Nucl. Instr, and Meth. 145 (1977) 555.

[25] R. Talman, Nucl. Instr. and Meth. 159 (1979) 189.

[26] A.H. Walenta et al., Nucl. Instr. and Meth. 161 (1979) 45; A.H. Walenta, Phys. Scripta 23 (1981) 354.

[27] W.W.M. Allison and J.H. Cobb, Ann. Rev. Nucl. Part. Sci. $30(1980) 253$.

[28] J. Vavra et al., Nucl. Instr. and Meth. 203 (1982) 109

[29] I. Lehraus et al., IEEE Trans. Nucl. Sci. NS-30 (1983) 50.

[30] R.S. Sternheimer and R.F. Peierls, Phys. Rev. B3 (1971) 3681:

R.S. Sternheimer et al.. BNL 31435 (1982).

[31] I. Lehraus et al., Nucl. Instr. and Meth. 196 (1982) 361.

[32] W.W.M. Allison, Phys. Scripta 23 (1981) 348

[33] I. Lehraus et al., Phys. Scripta 23 (1981) 727.

[34] I. Lehraus et al., Nucl. Instr. and Meth. 200 (1982) 199.

[35] A. Wagner. Proc. Int. Conf. on Instrumentation for colliding beam physics, SLAC-250 (1982) p. 76.

[36] H. Haseman, Ibid., p. 80

[37] B. Hallgren and H. Verweij, IEEE Trans. Nucl. Sci. NS-27 (1980) 333.

[38] T. Ludlam et al., IEEE Trans. Nucl, Sci. NS-28 (1981) 439

[39] T. Oshima et al., Proc. Int. Conf. on Instrumentation for colliding beam physics, SLAC-250 (1982) p. 101; R. Arai et al., KEK-P-82-24 (1982), submitted to Nucl Instr. and Meth.

[40] T. Ludlam et al., Proc. Int. Conf. on Instrumentation for colliding beam physics, SLAC-250 (1982) p. 96.

[41] I. Lehraus et al., IEEE Trans. Nucl. Sci. NS-30 (1983) 44

[42] J. Anderhub et al., Nucl. Instr. and Meth. 166 (1979) 581 
[43] J. Bourotte and B. Sadoulet, Nucl. Instr. and Meth. 173 (1980) 463.

[44] H.J. Hilke, Nucl. Instr. and Meth. 174 (1980) 145.

[45] H. Anderhub et al., Nucl, Instr. and Meth. 176 (1980) 323.

[46] M. Desalvo and R. Desalvo, Nucl. Instr. and Meth. 201 (1982) 357.
[47] J.C. Guo et al., Nucl. Instr. and Meth. 204 (1982) 77.

[48] D. Brozzi et al., CERN-UA1-TN 82/14 (1982);

C. Cochet et al., CERN-UA1-TN 82/17 (1982);

T. Markiewicz et al., CERN-UAl-TN 82/20 (1982).

[49] J. Vavra, IEEE Trans. Nucl. Sci. NS-30 (1983) 82

[50] P. Nemethy et al., Nucl. Instr. and Meth. 212 (1983) 273. 